\title{
Don't harm your patient - safety of bronchoscopy
}

\author{
F.J.F. Herth
}

Monaldi Arch Chest Dis 2009; 71: 1, 1-2.

Keywords: Fiberoptic bronchoscopy, Safety, side effects, Bronchial biopsies, Transbronchial biopsies, Bronchoalveolar lavage.

Department of Pneumology and Critical Care Medicine, Thoraxklinik, University of Heidelberg, Heidelberg, Germany.

Correspondence: Prof. Felix JF Herth, Chief, Department of Pneumology and Critical Care Medicine, Thoraxklinik, University of Heidelberg, Amalienstr. 5,D-69126 Heidelberg, Germany; e-mail: Felix.Herth@thoraxklinik-heidelberg.de

It is now more than 100 years since Killian introduced the rigid bronchscopy and 40 years since Ikeda [1] introduced the technique of flexible fibreoptic bronchoscopy, and this procedure has been established throughout the world.

Flexible bronchoscopy (FBS) with topical anaesthesia has been shown to be safer than rigid bronchoscopy [2]. Risks associated with bronchoscopy can be classified into those related to conscious sedation and medications and those related to the procedure itself. Risks from conscious sedation and other medications used during the procedure include medication-related cardiac arryhtmia, hypotension, hypoxaemia, unconsciousness and multiple other problems [3]. Risks associated with the procedure itself generally include respiratory depression, pneumonia, pneumothorax, airway obstruction, cardiorespiratory arrest, arrhythmias, and pulmonary oedema. Minor non-life threatening complications include, in order of frequency, vaso-vagal reactions, fever, cardiac arrhythmias, haemorrhage, airway obstruction, pneumothorax, nausea and vomiting [4].

Major complications such as bleeding, respiratory depression, cardiorespiratory arrest, arrhythmia, and pneumothorax occur in around $1-2 \%$ of cases. Mortality is rare, with a reported death rate of 0 to $0.04 \%$ [3, 4]. Most potential complications of rigid bronchoscopy can be avoided. These include injury to the teeth or gums, tracheal or bronchial tears, or severe bleeding. Complication rates should be below $0.1 \%$. Procedure-related mortality is also rare [5].

Unfortunately, studies on this topic are very few in literature, at least there are no controlled studies of the factors which may make a patient unfit for bronchoscopy so a decision to carry out the procedure is a balance between the likely benefit of obtaining diagnostic material (including therapeutic benefit) and an assessment of the likely risk in that individual patient.

Creedle [6] published the first retrospective study of the complications that occur in bronchoscopy, showing a mortality rate of $0.1 \%$ and an incidence of complications greater than $0.8 \%$. Suratt [7] and Peirera [8] published quiet similar results, again in a retrospective way.

But since a couple of years, no additional information about the topic was available. Nicola Facciolongo [9] published in the recent issue the experience of nineteen Italian centers. From $01 / 02 / 2002$ to $01 / 02 / 2003$ centers participated in the study by compiling a chart via the website with access reserved for each participating center. The sample was divided into two categories: the first group of explorative bronchoscopies which involve only endoscopic exploration and bronchoaspirates without other interventional procedures; the second group of non-explorative bronchoscopies which comprise endoscopic exploration associated with interventional procedures like bronchial biopsies (BB), transbronchial biopsies (TBB), transbronchial needle aspirates (TBNA), broncho-alveolar lavage (BAL), therapeutic bronchoscopies (TB) (Nd Yag laser, argon plasma, stent positioning, electrocautery etc.).

Overall a total of 20,986 bronchoscopies and 1.091 therapeutic bronchoscopies were reported. The total complications recorded were 227 (1.1\%), including $20(0.1 \%)$ during local anesthesia and pre-medication, $195(0.92 \%)$ during the endoscopic procedures and $12(0.05 \%)$ in the two hours following FBS. The total number of deaths was 4 $(0.02 \%)$. In relation to the type of procedure the diagnostic procedure most affected by complications is found to be TBB (3.4\%), while the one least affected by complications is explorative bronchoscopy $0.4 \%$. In therapeutic bronchoscopy, we found a $2.84 \%$ incidence of complications. The authors summarized, that bronchoscopy is a safe method with low incidence of mortality and complications. The preparation, experience and continuous training of the operators of the medical and nursing team seem to play a fundamental role in reducing the incidence of complications at least in certain procedures such as $\mathrm{BB}$ and $\mathrm{TBB}$.

What is the impact of the Italian trial? First diagnostic bronchoscopy in trained hands is a safe 
procedure. Also in a prospective study low morbidity and mortality could be shown. Second, depending to the sampling technique the risk for our patients is calculable and and an assessment of the likely risk in the individual patient is possible. And third, every pulmonologist can now, based on a high evidence level decide, where the procedure should be performed. The explorative bronchoscopies which involve only endoscopic exploration can be done in a outpatient setting in a adequate facility, more harmful procedures should be done in specialised centers and settings.

\section{References}

1. Ikeda S. The flexible bronchofiberscope. Keio J Med 1968; 17: 1-16.

2. Harrison BDW. Guidelines for care during bronchoscopy. Thorax 1993; 48: 584-96.
3. Smyth CM, Stead RJ. Survey of flexible bronchoscopy in the United Kingdome. Eur Resipir J 2002; 19: 358463.

4. Honeybourne D, Babb J, Bowie P, et al. British Toracic Society guidelines on diagnostic flexible bronchoscopy. Thorax 2001; 56: i1-i21.

5. Cavaliere S, Venuta F, Foccoli P, Toninelli C, La Face B. Endoscopic treatement of malignant airway obstructions in 2,008 patients. Chest 1996; 110: 1536-42.

6. Creedle W, Smiddy J, Elliott R. Complication of fiberoptic bronchoscopy. Am Rev Respir Dis 1974; 109: 67-72.

7. Suratt P, Smiddy J, Gruber B. Deaths and complication associated with fiberoptic bronchoscopy. Chest 1976; 69: 747-751.

8. Pereira W, Kovnat D, Snider GL. A prospective cooperative study of complication following flexible fiberoptic bronchoscopy. Chest 1978; 73: 813-816.

9. Facciolongo N, Patelli M, Gasparini S, et al. Incidence of complications in bronchoscopy. Monadi Arch Chest Dis 2009; 71: 7-14.

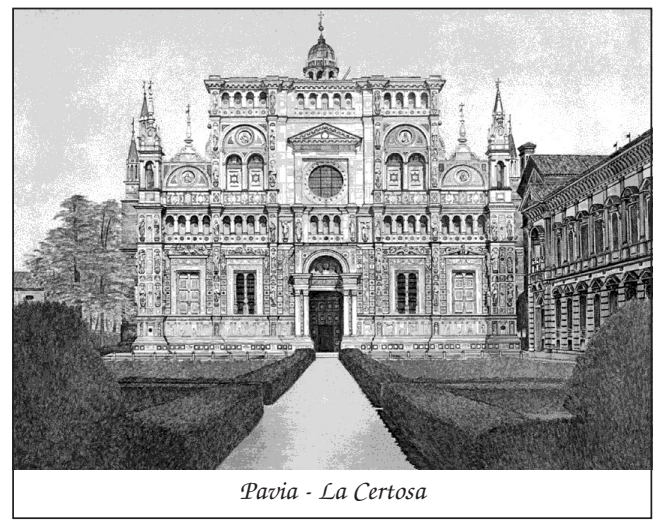

